\title{
Uma análise do desenvolvimento motor de crianças com transtorno do déficit de atenção e hiperatividade (TDAH)
}

\author{
Lidiane Aparecida Fernandes* \\ Débora Marques de Miranda** \\ Patrick Costa Ribeiro-Silva*** \\ Deyliane Aparecida Pereira**** \\ Mayndra Giusti Salvador***** \\ Guilherme Menezes Lage******
}

\section{Resumo}

O Transtorno do Déficit de Atenção com Hiperatividade (TDAH) é decorrente de alteraçôes em áreas do cérebro que implicam em alteraçôes no controle inibitório, na memória de trabalho, no tempo de reação, além de outras funçôes. As habilidades motoras globais e finas estão comprometidas nas crianças com TDAH e uma desordem ou desarranjo no desenvolvimento motor (DM) pode interferir em diversas fases do desenvolvimento global da criança. O objetivo do estudo foi analisar o DM de crianças com TDAH a partir da avaliação das habilidades motoras fundamentais. A amostra foi composta por oito crianças do sexo masculino com idade média de 10,25 anos. O teste de habilidades motoras foi realizado através do Test of Gross Motor Development (TGMD-2). Os resultados alcançados ficaram dentro do esperado e evidenciaram valores abaixo do esperado tanto para o subteste de atividades locomotoras como também para atividades manipulativas. As crianças com TDAH apresentaram um déficit motor acentuado nas habilidades de controle de objetos. A partir da análise do padrão de movimentos é possível identificar os fatores que tornam o movimento limitado, possibilitando a tomada de decisão sobre que habilidades e/ ou critérios motores devem ser enfatizados nos programas; o tempo de prática para cada habilidade e as metas de desempenho da criança. Os resultados evidenciam a necessidade de programas de atividades físicas regulares e sistematizados e permitem concluir que crianças diagnosticadas com TDAH apresentam um atraso no desempenho motor quando comparadas com crianças que estão de acordo com o que é esperado para a faixa etária.

Palavras-chave: Transtorno do déficit de atenção com hiperatividade;

Desenvolvimento motor; Coordenação motora grossa.

\footnotetext{
* Mestra em Ciências do Esporte pela Universidade Federal de Minas Gerais, Belo Horizonte, Minas Gerais, Brasil.

** Professora doutora da Universidade Federal de Minas Gerais, Belo Horizonte, Minas Gerais, Brasil.

*** Professor da Faculdade São Lourenço, São Lourenço, Minas Gerais, Brasil.

**** Doutoranda em Ciência da Nutrição pela Universidade Federal de Viçosa, Viçosa, Minas Gerais, Brasil.

***** Graduanda em Bacharelado em Educação Física pela Universidade Federal de Minas Gerais, Belo Horizonte, Minas Gerais, Brasil.

****** Professor doutor da Universidade Federal de Minas Gerais, Belo Horizonte, Minas Gerais, Brasil.
} 


\section{A children's motor development with analysis of disorder attention deficit hyperactivity disorder (ADHD)}

\section{Abstract}

The Attention Deficit Disorder with Hyperactivity (ADHD) is due to changes in brain areas involving a change in inhibitory control, working memory, reaction time, and other functions. Global and fine motor skills are impaired in children with $\mathrm{ADHD}$ and a disorder or breakdown in motor development (DM) can interfere in different phases of the overall development the child. The aim of the study was to analyze the DM of children with ADHD from the evaluation of fundamental motor skills. The sample consisted of eight male children with a mean age of 10.25 years, participants in a motor intervention project. The test motor skills was conducted through the Test of Gross Motor Development (TGMD-2). The results achieved were in line with expectations and showed values lower than expected for both the subtest of locomotor activity as well as for manipulative activities. Children with ADHD have a stronger motor deficits in object control skills. From the standard analysis of movement is possible to identify the factors that make limited movement, enabling decision making about what skills and / or motors criteria should be emphasized in the programs; the practice time for each skill, and the child's performance goals. The results highlight the need for regular and systematic physical activity programs. And allow us to conclude that children diagnosed with ADHD have a delay in motor performance when compared to children who are in agreement with what is expected for the age group.

Keywords: Attention deficit disorder with hyperactivity; Motor development; Motor coordination gross.

\section{Introdução}

A Síndrome reconhecida atualmente como Transtorno do Déficit de Atenção e Hiperatividade (TDAH) é uma das possibilidades diagnósticas quando o profissional encontra-se diante de queixas referentes ao comportamento discrepante daquele esperado para a faixa etária e que acarrete prejuízo para o desenvolvimento da criança em diferentes domínios da integraçáo social (PEREIRA, ARAÚJO, MATTOS, 2005). O TDAH é decorrente de alteraçôes em áreas do cérebro que implicam principalmente nos processos de aprendizagem, concentração e ações motoras. As principais regióes afetadas são o córtex parietal e pré-frontal, o cerebelo, os gânglios da base e os circuitos associados, que implicam uma alteração no controle inibitório, na memória de trabalho, no tempo de reação, para além de outras funçôes de execução (BUSH; VALERA; SEIDMAN, 2005).

Segundo Spencer (2008) o TDAH é um distúrbio neurocomportamental que afeta principalmente crianças em idade escolar, podendo se estender na idade adulta. 
O período da alfabetização é o momento em que há uma superposição de habilidades para a ocorrência da aprendizagem da leitura e escrita. Essa aprendizagem envolve habilidades cognitivas, linguísticas e motoras que exigem dos escolares o uso dos componentes sensório-motores e perceptivos, ou seja, a capacidade de decodificação das palavras e a açáo motora adequada para a execução do ato motor da escrita (CAPELLINI; SOUZA, 2008).

Em relação ao sexo, o transtorno é mais comum nos meninos que nas meninas (BRAZELTON; SPARROW, 2003). Essa proporção pode variar de três a cinco meninos para cada menina o que pode ser observado no estudo de Szatmari et al. (1989), que identificaram prevalência de TDAH significativamente maior em meninos $(9,0 \%)$ em comparação as meninas $(3,3 \%)$. A prevalência do transtorno é bastante variada, podendo ser observado em $3 \%$ a $9 \%$ da população de escolares. Ainda não há consenso na literatura sobre a sua causa específica, acreditando-se que pode estar relacionado a uma etiologia multifatorial dos quais os fatores genéticos e ambientais estão presentes (SILVA et al., 2012).

No que diz respeito aos comportamentos, crianças com TDAH tendem apresentar um padrão atípico de comportamentos quando comparados com crianças da mesma faixa etária. Estes comportamentos podem manifestar-se ao longo dos primeiros anos de vida, denominado tríade sintomatológica, destacando-se a desatenção, hiperatividade e impulsividade. Tais características, segundo Escobar et al. (2005) causam problemas no seu desenvolvimento em vários domínios relativos a integração social, a noção de tempo, a aquisição da linguagem e também ao desenvolvimento motor.

Complementando esta ideia, Poeta e Neto (2005) ressaltam que as dificuldades de aprendizagem e as perturbações motoras são manifestaçôes que acompanham o TDAH. No que concerne as perturbaçôes motoras, as habilidades motoras globais e finas estão comprometidas nas crianças com TDAH, especialmente em idade escolar (TONIOLO et al., 2009), por conseguinte o seu desenvolvimento motor é afetado e desencadeia incapacidades no desempenho das atividades diárias, por exemplo, grafia insatisfatória e deixar cair objetos.

Segundo Gallahue e Ozmun (2005), o desenvolvimento motor é um processo de contínua alteração no comportamento motor ao longo do ciclo da vida, proporcionada pela interação entre as necessidades da tarefa, a biologia do indivíduo e as condiçôes do ambiente (GALLAHUE; OZMUN, 2005). Ou seja, a interação destes fatores influencia diretamente as mudanças na capacidade motora do indivíduo, determinando a quantidade e a extensão da aquisição das destrezas motoras (VALENTINI; TOIGO, 2006).

As habilidades motoras fundamentais formam uma série de habilidades de locomoção, controle de objetos e de estabilização desenvolvidas na infância e consideradas importantes para o engajamento futuro em esportes, atividades de vida diária e atividades físicas em geral (GALLAHUE, 2005; STODDEN et al ., 2008). Estas habilidades, como o saltar, arremessar, correr e chutar, são resultado da interação entre o contexto de ensino, a motivaçáo, o desenvolvimento neurológico, as experiências 
passadas e as condiçóes socioculturais (VALENTINI; TOIGO, 2006). No entanto, embora o desenvolvimento motor infantil não ocorra de forma linear (SILVEIRA et al ., 2005), espera-se que a criança com idade escolar tenha tido oportunidade de praticar uma grande quantidade de habilidades motoras fundamentais e que estas estejam estruturadas em seu padrão maduro (VALENTINI; TOIGO, 2006). Contudo, sabe-se que pode haver diferenças individuais e que a idade cronológica não é o único fator influenciador do nível de desenvolvimento, por exemplo, se as crianças passarem pelo período crítico ${ }^{1}$ para o desenvolvimento das habilidades motoras sem conseguir aprimorá-las até o padráo maduro, podem ter maiores dificuldade no processo de aquisiçáo do padráo motor posteriormente. Desta forma, o próprio desenvolvimento da fase motora especializada pode ser prejudicado.

Para Gallahue (2005), as crianças com idades entre 8 e 12 anos se encontram num período crítico em que as habilidades motoras fundamentais devem ser refinadas e combinadas para que possam ser executadas no contexto esportivo (habilidades motoras especializadas). No entanto, alguns estudos avaliaram crianças com desenvolvimento típico e evidenciam que muitas apresentam atrasos no desenvolvimento motor esperado (VALENTINI, 2002a; FERREIRA et al., 2006; NOBRE, 2013). De forma similar, estudos que investigaram o desenvolvimento motor em crianças com desenvolvimento atípico (MEDINA-PAPST; MARQUES, 2010; TONIOLO et al., 2009) também encontraram atraso no padrão de movimento, ou seja, muitas das crianças nesta faixa etária não apresentam padrôes maduros nas habilidades motoras fundamentais. Decerto, uma desordem ou desarranjo no desenvolvimento motor pode interferir em diversas fases do desenvolvimento global da criança.

Diante do exposto, observa-se que crianças com TDAH tem predisposição a apresentarem comprometimentos nas habilidades motoras globais e finas. Dessa forma, o diagnóstico do desenvolvimento motor permite aos profissionais identificarem os fatores que tornam o movimento limitado, possibilitando a tomada de decisão sobre que habilidades e/ou critérios motores devem ser enfatizados em programas de intervençáo; o tempo de prática para cada habilidade motora, e as metas de desempenho da criança (VALENTINI; RUDISILL, 2004a; 2004b). É importante compreender todos os aspectos do desenvolvimento motor global de crianças com e sem transtornos, entendendo como o sistema de controle motor se auto-organiza e reage as demandas motoras. Logo, o presente estudo tem como objetivo analisar o desenvolvimento motor de crianças do sexo masculino com TDAH a partir da avaliação das habilidades motoras fundamentais.

\section{Metodologia}

\section{Amostra}

A seleção da amostra deste estudo foi por conveniência, sendo composta por oito crianças $(\mathrm{n}=8)$ do sexo masculino, com idade média de 10,25 $\pm 1,15$ anos, previamente diagnosticadas com TDAH pelo Núcleo de Investigaçóes sobre a Impulsividade e Atençáo (NITIDA) da Universidade Federal de Minas Gerais (UFMG) e participantes do projeto de extensão intitulado Intervençáo Motora para Crianças com Transtorno de Atenção e Hiperatividade (IMPACTAH) da UFMG (Tabela 1). 
A amostra foi composta por crianças que não realizavam atividades físicas em escolinhas de esportes e projetos de extensão os dados foram coletados no primeiro dia de intervenção motora das crianças participantes do projeto do projeto IMPACTAH. A coleta dos dados foi realizada no laboratório do Grupo de Desenvolvimento e Aprendizagem Motora (GEDAM), localizado na Escola de Educação Física, Fisioterapia e Terapia Ocupacional da Universidade Federal de Minas Gerais (CENESP/ EEFFTO/UFMG). Todos os responsáveis pelos voluntários assinaram um Termo de Consentimento Livre e Esclarecido (TCLE) e o Termo de Assentimento, sendo todos os procedimentos realizados conforme a Resolução do Conselho Nacional de Saúde (no 1931/2009) que descreve sobre pesquisas com seres humanos.

Tabela 1 - Características da amostra

\begin{tabular}{|c|c|c|c|}
\hline Nome & Idade Escolar & $\begin{array}{c}\text { Idade Biológica } \\
\text { Ano/Mês }\end{array}$ & $\begin{array}{c}\text { Data de } \\
\text { Nascimento }\end{array}$ \\
\hline Voluntário 1 & $5^{\text {o }}$ ano & 10,8 & $27 / 07 / 2003$ \\
\hline Voluntário 2 & $5^{\text {o }}$ ano & 10,7 & $14 / 06 / 2003$ \\
\hline Voluntário 3 & $5^{\circ}$ ano & 9,9 & $12 / 05 / 2004$ \\
\hline Voluntário 4 & $5^{\text {o } \text { ano }}$ & 9,5 & $18 / 08 / 2004$ \\
\hline Voluntário 5 & $4^{\text {o }}$ ano & 8,7 & $02 / 10 / 2005$ \\
\hline Voluntário 6 & $5^{\text {o }}$ ano & 8,5 & $02 / 08 / 2005$ \\
\hline Voluntário 7 & $1^{\text {o }}$ ano & 6,8 & $15 / 05 / 2007$ \\
\hline Voluntário 8 & $1^{\circ}$ ano & 6,7 & $31 / 08 / 2007$ \\
\hline
\end{tabular}

\section{Instrumento: test of gross motor development (TGMD - 2)}

O TGMD é um instrumento destinado a avaliar as habilidades motoras fundamentais de crianças. O teste foi proposto por Ulrich (2000) com a finalidade de avaliar o desenvolvimento motor global em crianças de 3 a 10 anos de idade, sendo um instrumento que apresenta confiabilidade e validade para as crianças brasileiras (VALENTINI, 2008).

O teste avalia 12 habilidades motoras fundamentais, das quais 6 são habilidades de locomoção (correr, galopar, saltitar, dar uma passada, saltar horizontalmente e correr lateralmente) e 6 são habilidades de controle de objetos (rebater, quicar, receber, chutar, arremessar por cima do ombro e rolar uma bola). Cada habilidade dos subtestes locomotor e controle de objetos possuem de três a cinco componentes comportamentais que são apresentados como critério de desempenho (ULRICH, 2000). Caso a execução observada apresente o critério de desempenho esperado, um ponto lhe é creditado. Ao final, ocorre o somatório dos pontos de duas tentativas, englobando todas as habilidades motoras, as quais indicam o nível de execução das habilidades no respectivo subteste, denominado de "escore bruto". A partir do escore 
bruto, é extraído das tabelas normativas do teste, o escore padrão em cada subteste. Essa medida é baseada no desempenho motor esperado de acordo com o sexo e faixa etária da criança que varia de três anos a dez anos e onze meses de idade. Existe também a possibilidade de obter o quociente motor amplo considerando-se o somatório dos escores padrão obtido por cada criança nos respectivos subtestes que é comparado aos dados normativos.

Segundo Ulrich (2000), o TGMD-2 pode ser utilizado para diagnosticar crianças que apresentam dificuldades no domínio das habilidades motoras fundamentais em relação aos seus pares, auxiliar no planejamento de um programa de intervenção para o desenvolvimento das habilidades motoras fundamentais, avaliar o progresso de crianças no desenvolvimento de habilidades motoras fundamentais e servir como instrumento em pesquisas envolvendo avaliaçáo do desenvolvimento motor. De modo geral, o TGMD-2 possibilita verificar o quanto a criança consegue realizar as tarefas que envolvem as principais habilidades motoras fundamentais e o desempenho com que as crianças coordenam os membros (superiores e inferiores) e o tronco durante a realização da tarefa motora.

Para cada habilidade são observados de 3 a 5 critérios motores específicos, permitindo uma avaliação separada de cada subteste. Para aplicação dos testes foram utilizados os seguintes materiais: 1 Câmera Digital da marca Sony modelo DCR -HC28; um tripé para câmera; uma bola de futebol, uma bola de vôlei; uma bola de tênis; um bastão; um suporte para uma bola média; fita métrica; e cones.

As tarefas do teste envolvem habilidades locomotoras e habilidades de controle de objeto.

Habilidades locomotoras:

1. Correr: O participante deverá realizar uma corrida em linha reta.

2. Galopar: O participante deverá executar um saltito com elevação de ambos os pés.

3. Saltitar: O participante deverá executar três saltitos com uma perna.

4. Dar uma passada: $\mathrm{O}$ participante deverá realizar um salto com extensão e troca de pernas.

5. Saltar horizontalmente: $\mathrm{O}$ participante deverá realizar um salto para frente com ambos os pés.

6. Correr lateralmente: $\mathrm{O}$ participante realizará um deslize lateral com afastamento das pernas seguidas de um saltito.

Habilidades controle de objetos:

1. Rebater: Com as duas mãos e com o auxílio de um taco e uma bolinha, o participante deverá golpear a bolinha lançada pelo avaliador. 
2. Quicar: Com o auxílio de uma bola média, o aluno deverá quicar a bola seguidamente.

3. Receber: $\mathrm{O}$ avaliador deverá lançar uma bola para o participante receber.

4. Chutar: $\mathrm{O}$ aluno deverá chutar uma bola parada.

5. Arremessar por cima do ombro: Com o auxílio de uma bola pequena, o aluno deverá lançá-la.

6. Rolar uma bola: O participante deverá rolar uma bola entre dois cones.

\section{Procedimentos}

O estudo foi realizado em fase única, no início da intervenção, na qual inicialmente os pais dos voluntários assinaram o Termo de Consentimento Livre e Esclarecido (TCLE), declarando conformidade com a participação no estudo. Foram obtidos os dados da criança como data de nascimento e a série escolar.

Inicialmente, todas as crianças da amostra foram convidadas a participar do estudo mediante autorização dos pais ou responsáveis. Em seguida, foi realizado a bateria de testes com a versão brasileira do TGDM-2. Para excluir possíveis variáveis intervenientes como estímulos distratores, excesso de sons e aspectos motivacionais, para o ambiente de realização dos testes foi utilizado um espaço no qual estavam presente apenas a avaliadora, um auxiliar e a criança participante. A avaliadora e o auxiliar fizeram um treinamento prévio para se familiarizarem com a aplicação do teste.

Todos os movimentos foram gravados em vídeo e, após as filmagens, os movimentos foram analisados a partir de critérios estabelecidos em lista de checagem para cada habilidade motora. Para o desempenho correto de um componente (critério) foi pontuado 1 ponto; ou 0 para o desempenho insatisfatório. Esse procedimento foi realizado para cada uma das duas tentativas e, em seguida, foi totalizado o escore obtido nas duas tentativas para obter um escore bruto da habilidade em cada item. A análise das filmagens foi feita separadamente por dois avaliadores experientes, na qual foi realizado o índice de concordância inter-observadores (THOMAS; NELSON; SILVERMAN, 2011) com percentual mínimo de oitenta e cinco (85\%) para garantir a qualidade de análise dos resultados. Nos casos raros de discordância entre os dois avaliadores, a avaliação da referida habilidade foi novamente realizada. Em todos os casos a identidade das crianças foi preservada, sendo elas identificadas por meio da numeração definida anteriormente à filmagem.

Os participantes foram instruídos sobre as tarefas a serem realizadas de forma padronizada e de acordo com o sugerido pelo protocolo do TGMD-2. Para cada habilidade os participantes receberam uma demonstração e instrução verbal até alcançar a compreensão da criança. Não foram ditas palavras motivacionais aos participantes que pudessem interferir na execução das tarefas. Foram realizadas três repetiçôes de cada habilidade motora, a primeira execuçáo conforme sugerida por Ulrich (2000) teve como objetivo verificar se a criança havia entendido a tarefa e duas outras repe- 
tições para análise dos dados, sendo computada a média das duas outras repetiçôes para análise dos dados.

\section{Análise dos dados}

Todos os subtestes foram avaliados de forma qualitativa do movimento e tiveram uma pontuação de 0 (zero) ou de 1 (um) ponto. Pontuação 0 (zero) para o aluno que não executou corretamente o movimento e 1 (um) para o aluno que executou o movimento corretamente. Os dados brutos foram obtidos através do somatório de pontos recebidos pelo indivíduo na execução de cada habilidade motora, considerando-se a forma do movimento executado pelo indivíduo em cada tentativa, sendo um total de três tentativas.

Escores padróes, que levam em consideração a idade da criança, foram usados neste estudo, e foram elaborados a partir dos dados brutos e da idade dos indivíduos, ou seja, se um determinado componente do movimento está presente na execução da habilidade motora ou não, dentro de uma idade média para o desenvolvimento deste componente. A amplitude de resultados é de 1 (um) a 20 (vinte) para cada subescala.

Os dados foram fornecidos através do software easy TGMD-2. Foi utilizado uma análise descritiva com medidas de desvio padrão e média. Todos os procedimentos estatísticos foram realizados no pacote excel 2013.

\section{Resultados}

Para o subteste locomotor os resultados evidenciaram que 50\% dos participantes apresentaram um resultado abaixo da média, $25 \%$ apresentou um resultado caracterizado como fraco e $12,5 \%$ como muito fraco quando classificados de acordo com o TGMD-2. Em contraste, apenas $12,5 \%$ dos participantes, ou seja, 1 participante apresentou resultado da avalição para o desenvolvimento motor dentro da média da amostra analisada (Tabela 2).

Para o escore bruto os participantes apresentaram uma média de 32,87 pontos sendo que os valores desta avaliaçáo se encontram entre 0 e 48 pontos. A média da idade equivalente dos participantes é de 5,37 anos e a idade de atraso motor para os participantes apresentou uma média de 2,75 anos. Estes resultados demonstram o atraso motor das crianças com TDAH quando comparados aos dados normativos.

Tabela 2 - Resultado do subteste locomotor

\begin{tabular}{|c|c|c|c|c|}
\hline Aluno & Escore Bruto & $\begin{array}{c}\text { Idade } \\
\text { Equivalente }\end{array}$ & Atraso Motor & $\begin{array}{c}\text { Avaliaçáo } \\
\text { Descritiva }\end{array}$ \\
\hline Voluntário 3 & 23 & $" 3-9 "$ & $" 6-0 "$ & Muito fraco \\
\hline Voluntário 2 & 34 & $" 5-6 "$ & $" 5-1 "$ & Fraco \\
\hline Voluntário 5 & 33 & $4-6 "$ & $43-1 "$ & Fraco \\
\hline
\end{tabular}


Continuação da Tabela 2

\begin{tabular}{|c|c|c|c|c|}
\hline Voluntário 4 & 37 & "6-0" & "3-5" & $\begin{array}{c}\text { Abaixo da } \\
\text { Média }\end{array}$ \\
\hline Voluntário 6 & 36 & "6-0" & "2-5" & $\begin{array}{c}\text { Abaixo da } \\
\text { Média }\end{array}$ \\
\hline Voluntário 7 & 27 & "4-3" & "2-4" & $\begin{array}{c}\text { Abaixo da } \\
\text { Média }\end{array}$ \\
\hline Voluntário 8 & 29 & "4-9" & "1-10" & $\begin{array}{c}\text { Abaixo da } \\
\text { Média }\end{array}$ \\
\hline Voluntário 1 & 44 & "10-0" & "0-8" & Na média \\
\hline Média & 32,88 & "5-37" & \multicolumn{2}{|c|}{ "2-75" } \\
\hline Desvio Padráo & $\pm 6,53$ & \pm "1-87" & \multicolumn{2}{|c|}{ \pm "1-80" } \\
\hline
\end{tabular}

Em relaçáo aos valores do subteste de controle de objetos todos os participantes apresentaram uma avaliação do desenvolvimento motor muito fraca (Tabela 4). A média do escore bruto foi de 20,12 pontos sendo que os valores desta avaliaçáo se encontram entre 0 e 44 pontos. A média da idade equivalente dos participantes foi de 3,37 anos e a média do atraso motor foi de 4,75 anos.

Os resultados do subteste de controle de objetos apresentaram um atraso motor ainda mais evidente com uma diferença de 2 anos a mais de atraso que o subteste de locomoção. Os valores do escore total também apresentaram resultados inferiores quando comparados com os resultados do subteste de controle de objetos, com uma diferença de 12,75 pontos. Estes resultados apontam que o desempenho de crianças com TDAH apresentam um déficit motor acentuado nas habilidades fundamentais e que para as habilidades de controle de objetos este déficit é ainda maior que as habilidades de locomoção motora.

Tabela 3 - Tabela do subteste de controle de objetos

\begin{tabular}{|l|c|c|c|c|}
\hline Aluno & Escore Bruto & $\begin{array}{c}\text { Idade } \\
\text { Equivalente }\end{array}$ & Atraso Motor & $\begin{array}{c}\text { Avaliação } \\
\text { Descritiva }\end{array}$ \\
\hline Voluntário 2 & 18 & $"<3-0 "$ & $" 7-8 "$ & Muito fraco \\
\hline Voluntário 4 & 16 & $"<3-0 "$ & $" 6-6 "$ & Muito fraco \\
\hline Voluntário 6 & 26 & $" 4-6 "$ & $" 3-11 "$ & Muito fraco \\
\hline Voluntário 1 & 23 & $" 4-0 "$ & $" 6-8 "$ & Muito fraco \\
\hline Voluntário 3 & 27 & $" 4-9 "$ & $" 5-0 "$ & Muito fraco \\
\hline Voluntário 7 & 16 & $"<3-0 "$ & $" 3-8 "$ & Muito fraco \\
\hline Voluntário 5 & 20 & $43-3 "$ & $4-4 "$ & Muito fraco \\
\hline Voluntário 8 & 15 & $"<3-0 "$ & $4-8 "$ & Muito fraco \\
\hline Média & $\mathbf{2 0 , 1 3}$ & $" 3-37 "$ & \multicolumn{2}{|c|}{$" 4-75 "$} \\
\hline Desvio Padráo & $\pm 7,70$ & \pm "0-79" & \multicolumn{2}{|c|}{ \pm "1-67" } \\
\hline
\end{tabular}




\section{Discussão}

O presente estudo teve como objetivo analisar o desenvolvimento motor de crianças com TDAH a partir da avaliação das habilidades motoras fundamentais. Para isso, utilizou-se uma bateria de testes que avalia o nível de desenvolvimento motor global a partir da análise do padrão de movimento das habilidades de locomoção e de controle de objetos. Através do TGMD-2 foi possível observar que todas as crianças do estudo apresentaram um desempenho inferior ao esperado no subteste locomotor e também no subteste de controle de objetos o que corrobora com estudos anteriores (LOPES, 2013; BRANCO, 2013, TONIOLO et al., 2009). Podemos sugerir que o déficit no desenvolvimento motor destas crianças a dois fatores principais, sendo eles resultantes do distúrbio neurocomportamental e da escassez de oportunidades de interação e desenvolvimento destas habilidades na escola e no convívio social.

Para crianças com TDAH verifica-se um padrão típico de comportamentos que podem manifestar-se ao longo dos primeiros anos de vida, destacando-se a desatenção e hiperatividade-impulsividade. Esta tríade sintomatológica desencadeia um comportamento discrepante, de acordo com o que é esperado para a faixa etária, causando problemas no seu desenvolvimento em vários domínios relativos à integração social, a noção de tempo, a aquisição da linguagem e também ao desenvolvimento motor (ESCOBAR et al., 2005). Estas habilidades podem ser consideradas como alicerces para o desenvolvimento e aprendizagem de habilidades mais complexas (TANI et al ., 2010), demonstrando uma forte associação com o engajamento em atividades físicas ao longo da vida (STODDEN et al ., 2008).

Essa tendência de atraso no desenvolvimento motor é confirmada pela literatura especializada que aponta que aproximadamente $50 \%$ dos escolares com problemas de atençáo sáo identificados concomitantemente com uma desordem do desenvolvimento da coordenação, o que acaba comprometendo a realização de tarefas motoras (PEREIRA, ARAÚJO, MATTOS, 2005). Essas informaçôes são relevantes pelo fato de mostrarem a importância das avaliações motoras nas crianças com TDAH. Nos casos em que forem identificadas dificuldades motoras deve-se oportunizar intervençôes motoras, visando atenuar essas dificuldades e permitir uma melhor qualidade de movimento.

Outro resultado evidente neste estudo foi a diferença no desempenho dos subtestes. Para as habilidades do subteste de controle de objetos os participantes apresentaram um desempenho inferior quando comparado com os resultados do subteste de locomoção. Estes resultados mostram que as crianças com TDAH deste estudo apresentam um déficit ainda maior no desenvolvimento das habilidades manipulativas como rebater, quicar, receber, chutar, arremessar por cima do ombro e rolar uma bola. Sugerimos novos trabalhos de investigação do desenvolvimento motor das habilidades fundamentais de crianças com TDAH que possam investigar se o déficit acentuado nas habilidades de controle de objetos se repete em maiores amostras ou se é uma particularidade deste grupo de crianças que foram analisadas.

As crianças com desenvolvimento motor atípico, ou que apresentam risco de atrasos motores, merecem atenção e açôes específicas, já que os problemas de coorde- 
nação e controle do movimento poderão se prolongar até a fase adulta (CANTELL; SMYTH; AHONEN, 2003). Além disso, os problemas associados ao transtorno podem desencadear uma série de prejuízos no desenvolvimento global da criança. Portanto, é fundamental que se ofereça a criança um ambiente diversificado, de situaçôes novas e que propicie meios diversos de resolução de problemas, uma vez que o movimento se apresenta e se aprimora por meio dessa interação, das mudanças individuais com o ambiente e a tarefa motora.

Diante do grande número de crianças em idade escolar identificadas com TDAH, pesquisas de diagnóstico e de problemas do desenvolvimento associados ao transtorno sâo importantes para que se possa melhor compreender e atender às necessidades apresentadas pelos escolares (SILVA et al., 2012). Sabendo da possibilidade do TDAH estar associado a dificuldades motoras e da importância das avaliaçóes motoras para identificação dessas dificuldades, o diagnóstico do desenvolvimento motor permite aos profissionais identificarem os fatores que tornam o movimento limitado, possibilitando a tomada de decisão sobre que habilidades e/ou critérios motores devem ser enfatizados nos programas; o tempo de prática para cada habilidade, e as metas de desempenho da criança (VALENTINI; RUDISILL, 2004a; 2004b). A identificação de níveis de desenvolvimento e funcionalidade de crianças é essencial para o desenvolvimento de programas interventivos que tenham como finalidade potencializar o desenvolvimento de novas habilidades, remediar as dificuldades já estabelecidas e/ou desenvolver novas estratégias de movimento (KING-THOMAS, 1987).

É fundamental que profissionais da área da saúde e da educação, em especial psicólogos, pedagogos e professores de educação física, atentem para a importância das avaliaçôes e implementação de atividades voltadas à essa população no ambiente escolar e para a relevância de intervençóes multidisciplinares, visando aumentar as possibilidades de obter resultados positivos no tratamento de crianças com Déficit de Atenção/Hiperatividade (SILVA et al., 2012).

Os resultados do presente estudo permitem concluir que crianças do sexo masculino diagnosticadas com TDAH apresentam um atraso no desempenho motor para o subteste locomotor e o subteste de controle de objetos quando comparadas com crianças que estão de acordo com o que é esperado para a faixa etária.

\section{Referências}

BRANCO, A. F. B.C. Desempenho manual e assimetria motora em crianças com perturbaçáo de hiperatividade com défice de atenção e com desenvolvimento típico. Estudo comparativo, numa tarefa de destreza manual final (Dissertação). Universidade do Porto, Porto, 2013.

BRAZELTON, T. B., SPARROW, J. D. 3 a 6 anos: momentos decisivos do desenvolvimento infantil. Porto Alegre: Artmed; 2003.

BUSH, G.; VALERA, E.; SEIDMAN, L. Functional neuroimaging of attention-deficit/ hyperactivity disorder: a review and suggested future directions. Biological Psychiatry, v. 57, n. 11, p. 1273 - 1284, 2005.

CANTELL, M. H.; SMYTH, M. M.; AHONEN, T. P. Two distinct pathways for developmental coordination disorder: Persistence and resolution. Human Movement Science, v. 22, p. 413-31, 2003.

CAPELLINI, A.S.; SOUZA, A.V. Avaliação da função motora fina, sensorial e perceptiva em escolares com dislexia. In: Sennye AL, Capovilla FC, Montiel, JM. Transtornos da aprendizagem da avaliaçáo à reabilitaçáo. Sáo Paulo: Artes Médicas, p. 55 - 64, 2008. 
ESCOBAR, R et al. Worse quality of life for children with newly diagnosed attention-deficit/hyperactivity disorder, compared with asthmatic and healthy children. Pediatrics, v. 116, n. 3, p. 364 - 369, 2005.

FERREIRA, C. R. et al. Análise do padrão fundamental de movimento em crianças de 3 a 8 anos de idade. Revista Motricidade, v. 2, p. 134 - 142, 2006.

GALlahUE, D. L; OZMUN, J. C. Compreendendo o Desenvolvimento Motor: bebês, crianças, adolescentes e adultos. São Paulo: Phorte, 2005.

KING-THOMAS L. A therapist's guide to Pediatric Assessment. Boston: Little Brown, 1987.

LOPES, D. R.S. Desempenho Manual e Assimetria Motora em crianças com Perturbaçáo de Hiperatividade com Défice de Atençáo e com Desenvolvimento Típico. Estudo Comparativo, numa tarefa de destreza manual global (Dissertação). Universidade do Porto, Porto, 2013.

MEDINA-PAPST, J.; MARQUES, I. Avaliação do desenvolvimento motor de crianças com dificuldades de aprendizagem. Revista Brasileira Cineantropometria e Desempenho Humano, v. 12, n. 1, p. 36 - 42, 2010.

NOBRE, F. S. S. Desenvolvimento motor em contexto: contribuiçóes do modelo bioecológico de desenvolvimento humano. 2013. Tese (Doutorado) Programa de Pós-Graduação em Ciência do Movimento Humano, Universidade Federal do Rio Grande do Sul, Porto Alegre, 2013.

PEREIRA, H.S.; ARAÚJO, A.P.Q.C.; MATTOS, P. Transtorno do déficit de atenção e hiperatividade (TDAH): aspectos relacionados à comorbidade com distúrbios da atividade motora. Revista Brasileira Saúde Materna. Infantil, v. 5, n. 4, p. 391 - 402, 2005.

POETA, L.; NETO, F. Intervenção motora em uma criança com transtorno do déficit desatenção/hiperatividade (TDAH). Educación Física y Deportes, Revista Digital, v. 10, n. 89, 2005.

SILVA, J.; et al. Desempenho motor de escolares com e sem Transtorno de Déficit de Atenção/Hiperatividade (TDAH). ConScientiae Saúde, v. 11, n. 1, p. 76 - 84, 2012.

SILVEIRA, C. R. A. et al. Avaliação motora de pré-escolares: relaçôes entre idade motora e idade cronológica. Educación Física y Deportes, Revista Digital, v. 10, n. 83, 2005.

SPENCER, T. Attention déficit/ hyperactivity disorder. In Ebert, M.; Loosen, P.; Nurcombe,B.; Leckman, J. (2 Ed.), Current Diagnosis Treatment Psychiatry. USA: McGraw-Hill Companies, 2008.

STODDEN, D. F. et al. A developmental perspective on the role of physical competence in physical activity: An emergent relationship. Quest, v. 60, p. 290 - 306, 2008.

SZATMARY P.; OFFORD, D. R.; BOYLE, M. H. Ontario child health study: prevalence of attention deficit disorder with hyperactivity. Journal of Child Psychology and Psychiatry, v. 30, n. 2, p. 219 - 230, 1989.

THOMAS, J.R.; NELSON, J.K.; SILVERMAN, S. Research Methods in Physical Activity. Champaign, IL: Human Kinetics Publishers, 2011.

TONIOLO, C. et al. Caracterizaçấo do desempenho motor em escolares com transtorno de déficit de atenção com hiperatividade. Revista de Psicopedagogia, v. 26, n. 79, p. 33 - 40, 2009.

ULRICH, D. A. The Test of Gross Motor Development, 2o ed. Austin, Texas: Prod-Ed., 2000.

VALENTINI, C. N. Teste de desenvolvimento motor grosso: Validade e consistência interna para uma população gaúcha. Revista Brasileira Cineantropometria e Desempenho Humano, v. 10, n. 4, p. 399 - 404, 2008.

A influência de uma intervenção motora no desempenho motor e na percepção de competência de crianças com atrasos motores. Revista Paulista de Educaçáo Física, v. 16, n. 1, p. 61 - 75, 2002 b.

Percepçôes de competência e desenvolvimento motor de meninos e meninas: um estudo transversal. Movimento, Porto Alegre, v. 8, n. 2, p. 51 - 62, 2002a.

VALENTINI, N. C.; RUDISILL, M.E. Motivational Climate, Motor Skill Development and perceived competence: two studies of developmentally delayed Kindergarten children. Journal of Teaching in Physical Education, v. 23, n. 3, p. $216-234,2004$ b.

$\mathrm{Na}$ inclusive Mastery Climate Intervention on the Motor Skill Development of children. Adapted Physical Activity Quarterly, v. 21, n. 4, p. 330 - 347, 2004 a.

VALENTINI, N. C.; TOIGO, A. M. Ensinando Educaçáo Física nas séries iniciais: desafios e estratégias. 2. ed. Canoas: Salles, 2006. 
Uma análise do desenvolvimento motor de crianças com transtorno do déficit de atenção e hiperatividade (TDAH)

\section{Notas}

${ }^{1}$ Entende-se por período de tempo durante o qual um indivíduo é mais susceptível a determinada influência externa.

\section{Correspondência}

Lidiane Aparecida Fernandes - Universidade Federal de Minas Gerais, Escola de Educação Física. Universidade Federal de Minas Gerais - Pampulha. CEP: 31270901 - Belo Horizonte, Minas Gerais, Brasil.

E-mail: lidianefernandes12@yahoo.com.br - menezeslage@gmail.com - patrickribeiro.mtc@hotmail.com deyliane.pereira@gmail.com - mayndra92@yahoo.com.br - menezeslage@gmail.com

Recebido em 26 de abril de 2016

Aprovado em 29 de agosto de 2016

Revista Educação Especial | v. 30 | n. 57 | p. 115-128 | jan./abr. 2017

Santa Maria

Disponível em: <http://www.ufsm.br/revistaeducacaoespecial> 
